\title{
VI. The effect of temperature and magnetization on the resistance of graphite
}

\section{David E. Roberts B.Sc.}

To cite this article: David E. Roberts B.Sc. (1913) VI. The effect of temperature and magnetization on the resistance of graphite , Philosophical Magazine Series 6, 26:151, 158-176, DOI: $10.1080 / 14786441308634960$

To link to this article: http://dx.doi.org/10.1080/14786441308634960

曲 Published online: 08 Apr 2009.

Submit your article to this journal

Џll Article views: 4

Q View related articles $\square$

Citing articles: 5 View citing articles 5 
recalls the emission theory developed by Newton, while the main features of the undulatory theory are conserved. But the emission theory definitely postulates the kinetic nature of the whole of the optical energy in a medium-in strict accordance with Fermat's law. We conclude, accordingly, that either theory leads to the same conclusion.

28. Again, the motion of the Faraday tubes, in a medium, which may be taken to be a fluid medium of some kind, recalls the "motion of hollow vortex rings in a fluid in which portions in rotational and irrotational motion are freely mixed together and which Lord Kelvin has called vortex sponge." Now it has been shown that the equation of propagation of a plane wave in such a medium is the same as the equation of propagation of luminous vibrations in the æther, and that a spiral vortex in such a medium would behave in the same way as a tube of electric force. If this view of the electromagnetic field is accepted, the energy of the field intrinsic and actual must necessarily be entirely kinetic.

29. Finally, whether we view an atom as a vortex ring or simply regard it as made up of corpuscles and ions in rapid rotation, a molecule must be regarded intrinsically as a conservative system, whose energy is entirely kinetic, on any theory which at all holds the field. As further, we have argued that vibratory energy is also entirely kinetic, we reach the broad generalization that every form of energy involves motion, æthereal, corpuscular, or molar.

VI. The Effect of Temperature and Magnetization on the Resistance of Graphite. By David E. RoberTs, B.Sc., Research Fellow of the University of Wales *.

Notation.

$\theta=$ Temperature.

$\mathfrak{\$}=$ Intensity of magnetic field.

$\mathbf{R}_{0}=$ Resistance in zero field at $0^{\circ}$.

$\mathrm{R}^{\prime}=$ Resistance in zero field at $\theta^{\circ}$.

$\mathrm{R}^{\prime}=$ Resistance in field $\mathfrak{S}$ at $\theta^{\circ}$.

A, $a=$ Empirical constants.

$m=$ Exponential constant.

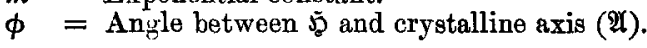

THE investigations of Grunmach and Weidert*, Patterson + , and others, on the effect of transverse magnetization on the electrical resistanee of various substances, show that paramagnetic and diamagnetic metals

* Communicated by Dr. H. du Bois. A preliminary publication appeared in Proc. Roy. Acad. Amst. xxi. p. 221 (1912).

$\dagger$ L. Grunmach and F. Weidert, Ann. der Phys. xxii. p. 141 (1907).

$\ddagger$ J. Patterson, Phil. Mag. [6] iii. p. 643 (1902). 
exhibit an increase of resistance when magnetized, while the three ferromagnetic metals, at least in sufficiently strong transverse fields, show a decrease. Although, as yet, no simple relation has been found to exist between the order of magnitude of this effect and the corresponding magnetic susceptibility, it may be noticed that the effect is a hundred times greater for diamagnetic cadmium than for paramagnetic tantalium and again a thousand times greater for bismuth. This element, as is well known, possesses rather a high diamagnetic specific susceptibility $\left(-1 \cdot 40 \cdot 10^{-6}\right)$. Soon after Morris Owen* found Ceylon graphite to show the highest susceptibility yet observed, Dr. W. J. de Haas was led by analogy to anticipate that graphite might exhibit a variation of resistance of an even higher order when magnetizel, and suggested to me to search for the effect. The preliminary experiments $\dagger$ performed with powdered graphite pressed into a thin plate, with irregularly shaped pieces, and with ordinary pencils, amply satisfied expertation, and justified an extended investigation of the phenomenon. Graphite crystallizes in the hexagonal rhombohedral (or according to the new notation, the ditrigonal scalenohedral) form; it.was once assigned to the monoclinic class of crystals, but latterly this view appears to have little support. Fairly well-defined crystalline pieces are, with few exceptions, only found in Pargas (Finland) and Ticonderoga (Now York); of the former I succeeded in obtaining a few specimens. Most frequently graphite is found filling veins, or in compact laminated masses, or as isolated flakes or scales embedded in the older crystalline rocks ; it posssesses perfect basal cleavage, and can thus be obtained in thin plates or flakes which are soft and flexible but not elastic.

Its density appears to be very variable. Le Chatelier and Wologdin $\ddagger$ have systematically investigated artificial graphite made after Acheson and that obtained from castiron, as well as native graphite from Scharzbach and Mograu (Bohemia), Siberia, Ceylon, and Australia. The density varied between $1 \cdot 6$ and $2 \cdot 2$; after the graphite had been purified by solutions of potash, \&c., and subjected to pressure, all the samples agreed in giving a density of $2.255\left(\mathrm{H}_{2} \mathrm{O}\right.$ at $4^{\circ}$ C.). Lime, silicic acid, iron, and carburetted hydrogen

* Morris Owen, Versl. Afd. Natuurkd. xx. p. 673 (1911); Ann. der Physik, xxxvii. p. 657 (1912).

$\dagger$ When transversely magnetized in a field of $20 \mathrm{kgs}$. the compressed Ceylon graphite gave an increase of resistance of 52 per cent.; an irregularly-shaped piece gave an increase of 219 per cent. ; HB and SB pencils by A. W. Faber gave only a 3 per cent. increase. The best sample GI. 15 gave as much as 400 per cent.

$\ddagger$ H. le Chatelier and S. Wologdin, Compt. Rend. cxlvi. p. 49 (1908). 
occur in minute quantities as impurities; many varieties contain as much as 99 per cent. of pure carbon. The chemical structure of graphite is probably a complicated one, but above $370^{\circ} \mathrm{C}$. graphite is the most stable of the three allotropic modifications of carbon. Its optical constants were lately determined by Zakrewski*.

Several properties of graphite have been investigated by Koenigsberger $\dagger$ and his co-workers; its conductivity for heat along its base-plane and also at right angles to its axis was found to have a very high value, viz., 0.85 (cal. $/ \mathrm{cm}$. sec. ${ }^{\circ} \mathrm{C}$.), which nearly equals that of copper $(0.9)$; this does not, however, at all agree with the law of Wiedemann and Franz.

They further (l.c.) investigated its thermoelectric effect (compared with copper) and. its Thomson effect, but found here no extreme values.

According to a private communication kindly sent to me by Prof. Koenigsberger, Gottstein $\ddagger$ found for the Hall effect in the variety of graphite which I term below G V., $R=-0.71$. In the case of variety $I$., which I preferably worked with, the Hall effect was found in an experiment by Washburne to be of the same order, but showed marked dissymmetry.

The following values of the specific resistance of graphite were found by Muraoka, Artom, Piesch, Koenigsberger and Weiss \$:-

Ceylon graphite at $0^{\circ} \ldots \ldots . .12 .10^{-4} \mathrm{ohm}$ per c.c.

Siberian graphite at $0^{\circ} \ldots \ldots . . \quad 11 \cdot 10^{-4}$, , ,

Greenland graphite at $15^{\circ} \ldots \quad 4.10^{-4} \quad, \quad$,

Ceylon graphite at $16^{\circ} \ldots \ldots \ldots .63 \cdot 6 \cdot 10^{-4}, "$,

On the other hand, my best graphite pieces of the variety GI. gave a specific resistance as low as $0 \cdot 5 \cdot 10^{-4}$, i. e. almost half that of mercury $\left(0^{\circ} 96 \cdot 10^{-4}\right.$ at $\left.18^{\circ}\right)$. This material also, at ordinary temperatures, showed an increase of resistance of +0.001 per degree rise of temperature, while at high and low temperatures the increase was +0.0025 per degree

* K. Zakrewski, Bull. Int. Acad. Cracow, (A) xxii. p. 122 (1910).

$\dagger$ J. Koenigsberger and J. Weiss, Ann. d. Phys. xxxv. p. 27 (1911); Verh. d. Phys. Ges. xiv. p. 9 (1912); J. Weiss, Inaug.-Diss., Freiburg i. B. $(1910)$.

I This will soon be published in a Dissertation.

5 Cf. Handb. d. Anorg. Chem. iij. (2 Abt.) p. 54 (1909); J .Koenigsberger and J. Weise, Ann. d. Pys. xxxv. p. 2 (1911). 
(cf. fig. 3). On the other hand, Koenigsberger and Reichenheim * found a decrease of resistance of one half by a rise of temperature from $-185^{\circ}$ to $+191^{\circ}$. According to Clay $\dagger$, the resistance of a glow-lamp filament decreases by 24 per cent. on heating it from $-255^{\circ}$ to $0^{\circ}$. The specific resistance of amorphous carbon has always been found to be much greater and a decrease accompanies increase of temperature; the temperature coefficient becomes numerically smaller as the change into the graphite modification proceeds, but no change in sign has previously been noticed $\ddagger$. Such a phenomenon has recently been observed by von Pirani $\S$ in the case of the so-called metallized filaments. Thick filaments, free from ash, carbonized twice above $2500^{\circ}$, showed finally the smallest specific resistance and a small positive temperature coefficient +0.00007 , which is exceeded by the above (variety GI.) thirty-five times. From this it appears possible to obtain preparations, having a vanishingly small temperature coefficient, which could be used as standard resistances $\|$.

The effect of magnetization on the resistance of graphite was investigated by Patterson (loc. cit.), who found the resistance of a glow-lamp filament increased by 0.027 per cent. in a transverse field of $25 \mathrm{kgss}$. Laws 9 has also investigated the effect for glow-lamp filaments, pencils, and for graphite, without, however, finding it to be of an exceptionally high order. At ordinary temperatures he found the increase of resistance of graphite in a transverse field of $11 \mathrm{kgss}$. to be only 1 per cent. of the resietance outside the field, while at the temperature of liquid air the effect appeared to be increased threefold. Within the small limits of his field it was found that the increase of resistance was proportional to the square of the field, and between the temperatures $-186^{\circ}$ and $+18^{\circ}$, inversely proportional to the absolute temperature. From what follows it will be seen

* J. Koenigsberger and O. Reichenheim, Physiz. Zeitsch. vii. p. 575 (1906).

+ J. Clay, Inaug.-Diss., Leiden, 1908.

t. Cf. Fandb. d. Phys. iv. p. 380 (1905); G. Wiedemann, Elektrizität, i. p. $539(1882)$.

(5npublished communication, from Dr. M. v. Pirani to Prof. Koenigsberger, and kindly sent to me by the latter.

II Since my first communication, Versl. Kon. Akad. Wet. Amst. xxi. p. 221 (1912), this suggestion has been made by H. Kost, Physik. Zeitschr, xiii. p. 896 (1912).

T S. C. Laws, Phil. Mag. [6] xix. p. 694 (1910). He investigated graphite from the Morgan Crucible Co., London.

Phil. Mag. S. 6. Vol. 26. No. 151. July 1913. 


\section{$162 \mathrm{Mr}$. D. E. Roberts on the Effect of Temperature and}

that his results are not in agreement with those obtained with my specimens.

Regarding the resistance effect in the case of crystallized bismuth, von Everdingen * found that the resistance outside the field could be represented by an ellipsoid of conductivity, the axis of rotation of which bore to the rectangular axes the relations $\sqrt{3}: \sqrt{5}$. If the crystalline axis was placed to coincide with that of the field, an ellipsoid of revolution was again obtained with a slightly different relation betwer:n the axes. If the crystalline axis was placed perpendicular to the field, the ellipsoid in this case had three different axes with marked differences in their relation to one another ; for inclined positions similar results hold in accordance with the principle of superposition. The relation between the transverse and longitudinal effect has been investigated by Lenard $\dagger$ for a "filament" from compressed bismuth containing zinc and iron; the resistance for an alternating current was measured for different angles $\psi$ between the filament (referred to the direction of the measuring current) and the field, and the results represented by the purely empirical formula

$$
\frac{\mathrm{R}^{\prime}-\mathrm{R}}{\mathrm{R}}=\mathrm{A}+\mathrm{B} \cdot \sin ^{2} \psi-\mathrm{C} \sin ^{2} 2 \psi \cos 2 \psi .
$$

The influence of temperature and transverse magnetization on the resistance (for steady currents) of pure diamagnetic electrolytic bismuth and of ferromagnetic "pure nickel" (commercial) was investigated in detail in this lat,oratory by Blake ; his publication contains an extensive summary of the new literature on this subject.

The longitudinal effect in nickel was investigated simultaneously by du Bois ‡. Quite recently Kamerlingh Onnes and Beckmann $\$$ have published measurements on bismuth, copper, iron, gold, and palladium at temperatures below $-200^{\circ}$.

\section{Experimental Arrangement.}

The specimens most used in the investigation were prepared from the same commercial Ceylon graphite (afterwards

* E. v. Everdingen, Arch. néerl. [2] iv. p. 462 (1901).

+ P. L. Lenard, Wied. Ann. xxxix. p. $646(1890)$

† F. C. Blake, Ann. d. Phys. xxviii. p. 449 (1909); H. du Bois, Ferh. Ned. nat. Congress, xii. p. 128, Utrecht (1909); cf. E. A. Owtn, Phil. Mag. [6] xxi. p. 122 (1911) ; C. W. Heaps, Pbil. Mag. [6] xxii. p. 900 (1911), and xxiv. p. 813 (1912).

§ H. Kamerlingh Ounes and B. Beckmann, Versl. Kon. Akad. Wet. Amst. xxi. pp. 263, 478, 481, 881 (1912). 
referred to as $G I_{\text {.) }}$ as that used by $O$ wen in his researches on its thermo-magnetical properties; a chemical analysis gave 0.19 per cent. of iron for one of his samples. In addition, tests were also made on the following different kinds :-

II. Ceylon graphite from the Comptoir minéralogique, Geneva, for which I have to thank Prof. du Bois. It was easily cleavable but not of a uniform structure, so that the cleavage plane was not sufficiently definite.

III. Ceylon graphite from Himbuluwa having internally a fine granular structure; its surfaces were highly polished but left no mark when rubbed on paper. For this specimen I am indebted to Prof. Grünling *, Munich.

IV. Graphite from the Limberg mine, Pargas (Finland), consisting of crystallized flakes imbedded in limestone and easily cleavable parallel to their surface. Mr. Magnus Wegelius kindly sent me specimens of this kind.

$V$. Ceylon graphire, for which $I$ am indebted to Prof. Koenigsberger, Freiburg i. B., irregularly cleavable in all directions.

VI. Graphite, which had been used by Dr. Reichenheim, and which was also sent to me by Prof. Koenigsberger. It was not regularly cleavable although small plates, apparently crystalline, could be obtained.

Chemical tests for the presence of ferruginous and other impurities could not, up to the present, be carried out.

In the investigations on graphite $I$. short rectangular pieces $(7-10 \mathrm{~mm}$. long, $1-2 \mathrm{~mm}$. broad, and $0.1-0.5 \mathrm{~mm}$. thick) were obtained by careful cleavage, and those selected for investigation which appeared of most pronounced and uniform erystalline structure.

It was found only possible to prepare specimens cut parallel to the crystalline bases or clearage plane; suitable pieces with their lengths parallel to the crystalline axis could not be obtained.

For the determination of the effect of transverse magnetization they were, in general, supported in the field so that the cleavage planes were perpendicular to the lines of force, which were thus parallel to the crystalline axis. On supporting the pieces freely in a magnetic field, it was observed that they moved so that the crystallic axis set itself perpendicular to the field, this axis thus coinciding with the direction of maximum diamagnetic susceptibility, which according to $O$ wen may reach a value of -15 millionths.

* A. Grünling, Zeitschr.f. Krist. u. Mineral. sxxiii. p. 208 (1900). 
The resistance of the graphite specimens, both in and out of the fields, was determined by a potentiometer method*, being compared directly with known resistances $(0 \cdot 1-1 \cdot 0$ ohm). The current and potentiometer leads were soldered to the ends of the specimen investigated, which were previously coppered electrolytically. In the rotation experiments the four leads, which consisted of four insulated straight copper wires, about $30 \mathrm{~cm}$. long and bound together by silk so as to form a fairly rigid rod, were fixed inside a metal tube. This tube could be turned within a second shorter coaxial tube rigidly attached to the frame of the electromagnet used, and carried a coaxial graduated disk. The angle of rotation was read off directly by means of a fixed pointer. The copper leads lay along the axis of rotation of the apparatus, and for the determination of the variation of the transverse effect with orientation of the graphite in the field, the graphite was soldered to the leads with its length along that of the leads and, for the determination of the longitudinal effect, at right angles to this position. The current through the graphite during a series of measurements was varied between 2 and 0.5 milliamperes according to its resistance. In order to eliminate thermoelectric and other junction effects $\dagger$ the current in the main circuit, as well as the potentiometer connexions, were successively reversed. The changes of resistance involved being considerable, it was found necessary to adjust the sensitiveness of the potentiometer during a single series of readings ; this was initially sufficient to detect differences of $0.0001 \mathrm{ohm}$. Small irregular variations in the resistance of a particular specimen were observed after it was subjected to the action of magnetic fields or to widely different temperatures. This change, however, amounted in general to less than 1 per cent.; no evidence was ever found of any regular hysteresis with respect to the magnetic field or with temperature. Through the kindness of Prof. Hagen and Dr. Hoffmann, the resistance of specimen G I. 15-that used in the experiments at different temperatures-was redetermined at $18^{\circ} \mathrm{C}$. in the Phys. Tech. Reichsanstalt by means of Diesselhorst's "compensation apparatus" $\ddagger$ and a differential galvanometer: good agreement was found. Some of the preliminary measurements had been made with Wheatstone's bridge method, and when repeated potentiometrically, practically the same results were obtained.

* F. Kohlrausch, Prakt. Physik, ii. Auflage, p. 422 (1910).

+ These were discussed in detail by F. O. Blake, loc. cit. p. 465 .

+ H. Diesselhorst, Zeitschr. für Instr.kunde, xxvi. pp. 173,297 (1906); xxviii. pp. 1, 38 (1908). 
To obtain the magnetic fields the latest large type 1911 model of the du Bois half-ring electromagnet was used. To obtain the highest fields at ordinary temperatures special prism-shaped pole end-pieces (XV.) were generally used$12 \mathrm{~mm}$. Iong and $1.6 \mathrm{~mm}$. wide : with these parallel endpieces $0.7 \mathrm{~mm}$. apart and a pair of extra polar coils a field of 50 kilogauss could be easily attained; this proved practically uniform over an equatorial length of $4 \mathrm{~mm}$. and uniform within 1 per cent. over $8 \mathrm{~mm}$. For observations at low and high temperatures the same thermomagnetic arrangement (XI.) was used as that adopted by du Bois and Wills*. The polar distance was $1.5 \mathrm{~mm}$., the diameter of the frontal planes $7 \mathrm{~mm}$., and the semi-angle $56^{\circ}$; as the frontal planes were not sufficiently parallel the field proved uniform within about 1 per cent. only. For the determination of the transverse effect at ordinary temperatures at the lower fields, pole-shoes (VII.) (10-5 mm. apart) and then pole-end pieces VIII., with their frontal planes adjusted to be exactly parallel $(2 \mathrm{~mm}$. apart) were used, so that over the space occupied by the graphite a practically uniform field was obtained.

The uniformity of the field was tested by means of differential measurements with an exploring coil of only $1.3 \mathrm{~mm}$. in diameter. The magnetic fields were measured by means of an exploring coil and a ballistic-galvanometer in the usual way + . It was assumed that the fields were appreciably the same at all the temperatures used for a given current through the coils of the electromagnet $\neq$.

\section{Experiments at ordinary temperature $\left(18^{\circ} \mathrm{C}.\right)$.}

About twenty specimens of Ceylon graphite I. were investigated, which all gave variations of resistance of a very high order. The increase in a transverse field of $20 \mathrm{kilo-}$ gauss, e.g., varied between 300 and 500 per cent. of the resistance in zero field. Considering the difficulty of obtaining specimens of graphite of definite crystalline structure, and having regard to the impurities occurring in the natural substance, the variations in the magnitude of the effect are not surprising. About five specimens, which gave the greatest variation of resistance, were investigated more particularly;

* H. du Bois, Zeitschr. für Instr.kunde, xxxi. p. 369, fig. 9 (1911).

+ H. du Bois, 'The Magnetic Circuit in Theory and Practice,' p. 300. London, 1896.

† P. Weiss and H. Kamerlingh Onnes, Versl. Kon. Akad. Amst. xviii. p. $794(1910 \%$, find $2 \cdot 1$ per cent. for the increase of the saturation value on cooling from $+20^{\circ} \mathrm{C}$. to $-253^{\circ} \mathrm{C}$. 
by analogy with the well-known behaviour of more or less pure bismuth * the assumption appeared justifiable that these were more likely to be pure and perhaps of more uniform crystalline structure. Some of the specimens were supported free between thin mica or glass plates; when imbedded in sodium silicate, collodion, or Canada balsam allowed afterwards to solidify, they did not experience any change in the magnitude of their increase of resistance in the magnetic field, thus eliminating any doubts that the effects were due to bodily strains in the graphite. In the final experiments at different temperatures the graphite pieces were supported by thin flexible wires between thin mica plates, so as to avoid any strain due to possible expansion or contrastion. The specimens could be mounted with their connexions so that the total thickness amounted to less than $0.7 \mathrm{~mm}$., thus enabling them to be examined in fields up to 50 kilogauss. Some of the isothermal curves obtained for different specimens, transversely magnetized, at $18^{\circ} \mathrm{C}$., with the cleavage plane normal to the field, are shown in fig. 1. The curves are for the specimens:-

\begin{tabular}{|c|c|}
\hline Specimen. & Resistance $R_{0}$. \\
\hline G I. $\quad 4 \ldots \ldots \ldots \ldots \ldots \ldots \ldots$ & 0.043 olun \\
\hline G I. $10 \ldots \ldots \ldots \ldots \ldots \ldots \ldots \ldots \ldots$ & 0080 \\
\hline G I. $11 \ldots \ldots$. & 0.016 , \\
\hline G I. $12 \ldots \ldots$ & 0.043 \\
\hline G I. $15 \ldots \ldots \ldots \ldots$ & $0.032 "$ \\
\hline
\end{tabular}

Attempts to identify the curves with such equations as

$$
\frac{\mathrm{K}^{\prime}}{\overline{\mathrm{R}}_{0}}=\frac{\mathrm{R}}{\overline{\mathrm{R}}_{0}}+b \mathfrak{S}+c \mathfrak{G}^{2}+\ldots
$$

failed; it was found, however, that all the curves obtained at ordinary temperatures could, well within experimental errors, be represented by the formula

$$
\frac{\mathrm{R}^{\prime}}{\mathrm{R}_{0}}=\frac{\mathrm{R}}{\mathrm{R}_{0}}+\mathrm{A} \cdot \mathfrak{S}^{m} \ldots . \quad . \quad . \quad . \quad .
$$

Owing to the difficulty of determining the dimensions of

* F. C. Blake, Ann. der Physik, xxviii. p. 449 (1909). 
the specimens, it is unfortunately impossible to give their absolute specific resistance with any exactitude.

Fig. 1.

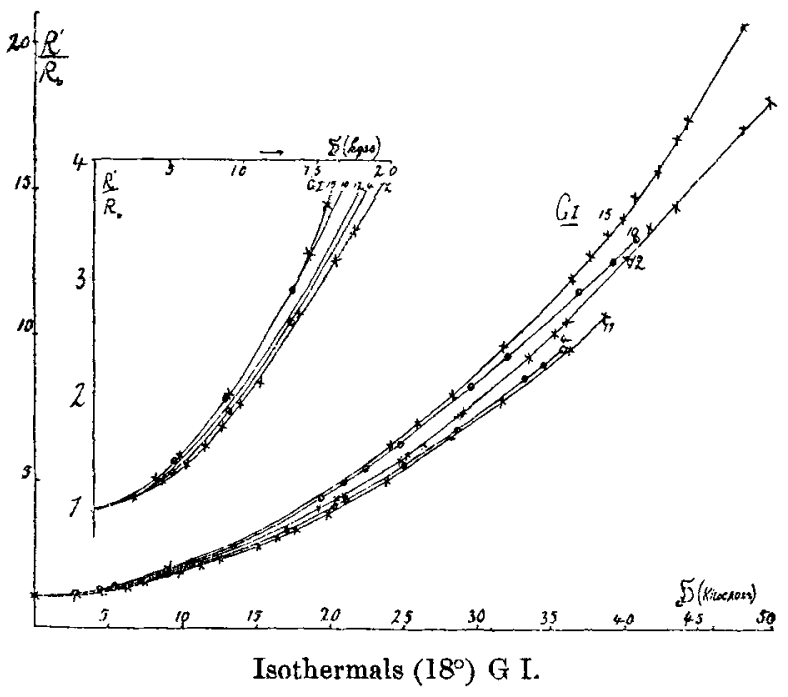

From equation (1) we have, taking logarithms

$$
\log \frac{\mathbf{R}^{\prime}-\mathrm{R}}{\mathbf{R}_{0}}=\log \mathrm{A}+m \log \mathfrak{S},
$$

which can be represented by a straight line if $A$ and $(m)$ are really constants, the coordinates being $\log \left(R^{\prime}-R\right) / R_{0}$ and $\log \mathfrak{j}$.

The values of $\log \left(R^{\prime}-R\right) / R_{0}$ and $\log \mathfrak{G}$, corresponding to the points on the curves shown in fig. 1 , when plotted were found to lie very nearly on straight lines practically parallel to one another, indicating that $m$ is the same constant for each of these specimens G I. 4, 10,11, 12, 15. In the catse of G I. 15-the one which gave an increase in resistance of the greatest order-equation (1) did not hold so well as for the other specimens, although the mean value of $m$ was the same for this as for the others. The values of $\mathrm{A}$ and $m$ obtained for the different specimens are given in Table I.

For each specimen $m=1 \cdot 7 t^{5 *}$.

* Within the experimental errors the exponent may also be $m=\sqrt{3}=1 \cdot 732$ or $m=7 / 4$. 
TABLE I.

Transrerse magnetization. Cleavage plane normal to the field.

\begin{tabular}{|c|c|c|c|c|}
\hline \multicolumn{5}{|c|}{ Isothermals at $18^{\circ} \mathrm{C}$. } \\
\hline \multirow{3}{*}{$\begin{array}{l}\text { Specimen. } \\
\text { G I. } 4 \ldots \ldots \ldots \ldots \\
\text { G I. } 10 \ldots \ldots \ldots \ldots\end{array}$} & $\mathbf{R}_{0}$ & \multicolumn{3}{|c|}{$\mathbf{R}^{\prime} / \mathbf{R}_{\mathbf{0}}=\mathbf{R} / \mathbf{R}_{0}+\mathbf{A} \mathfrak{S}^{m}$} \\
\hline & 0.0430 ohm & $1 \cdot 01$ & + & $0.0171 . \mathfrak{g}^{1 \cdot 745}$ \\
\hline & 0.0792, & 1.01 & + & $0.0205 . \mathfrak{S}^{1 \cdot 74^{5}}$ \\
\hline GI. $11 \ldots \ldots$ & 0.0162 & $1 \cdot 008$ & + & $0 \cdot 0162 . \mathfrak{S C}^{1 \cdot 74^{5}}$ \\
\hline G I. $12 \ldots$ & 0.0430 & $1 \cdot 014$ & + & $0 \cdot 0188 \cdot \mathfrak{S}^{1 \cdot 74^{5}}$ \\
\hline G I. $12 a \ldots$ & 0.0323 & $1 \cdot 01$ & + & $0 \cdot 0198 . \mathfrak{S}^{1 \cdot 74^{5}}$ \\
\hline GI. $15 \ldots \ldots \ldots \ldots$ & 0.0316 & $1 \cdot 02$ & + & $0 \cdot 0214 . \mathfrak{S}^{1 \cdot 745}$ \\
\hline
\end{tabular}

The results of the tests, which were made on the other kinds of graphite (see p. 163), which all showed the effect in a lesser degree or not at all, were as follows :-

GII. The best piece I conld prepare gave an increase of resistance of only 182 per cent. in a field of 20 kiloganss, the resistance out of the field being $0.0427 \mathrm{ohm}$.

G III. Different pieces of this graphite gave quite different results. A thin strip obtained from the rpper smooth and highly polished surface by cleavage, appeared on its underside of a fine granular structure. When transversely magnetized in a field of 20 kilogauss it showed an increase of resistance of 220 per cent., the resistance outside the field being $0.0786 \mathrm{ohm}$. A thin strip obtained from the underside of the same material, and having a high natural polish on both of its surfaces, gave the anomalous results. Its resistance outside the field was several hundred ohms, and diminished very rapidly with increase of temperature. In a magnetic field, however, no change in its resistance could be detected, while rough experiments indicated that it was paramagnetic. Three pieces similar to the latter were tested and agreed in giving these anomalous results.

G IV. The piece tested possessed a specific resistance of about $15.10^{-4} \mathrm{ohm}$ per $\mathrm{cm}^{3}$, i. e. of the same order as the average of $G I$. When transversely magnetized it showed an increase of resistance of 440 per cent. in a field of 26 kilogauss. Its initial resistance was $0.009 \mathrm{ohm}$. It appeared to be strongly diamagnetic. 
$G \mathrm{~V}$. The best piece of this kind which I could prepare gave a specific resistance of about $15 \cdot 6 \cdot 10^{-4} \mathrm{ohm}$ per cm..$^{3}$, and in a transverse field of 26 kilogauss it showed an increase of resistance of 163 per cent. It appeared to be diamagnetic.

G VI. Three pieces of this kind were tested, and these behaved similarly to graphite III. They possessed a very high specific resistance $\left(2 \cdot 14 \mathrm{ohm}\right.$ per $\left.\mathrm{cm} .{ }^{3}\right)$, and showed very marked decrease of resistance with increase of temperature (about 6 per cent. per degree). In a magnetic field of 26 kilogauss no change of resistance could be observed, although a change of 0.001 per cent. could have been detected. The material appeared to be strongly paramagnetic, which may be due to ferruginous impurities.

\section{Orientation of the Graphite in the Magnetic Field $\left(19^{\circ}-22^{\circ} \mathrm{C}\right.$. $)$.}

Preliminary observations with GI. 12 showed that in transverse fields the change of resistance of the graphite depended greatly on the angle $\phi$ between the crystalline axis $\mathfrak{U}$ and the magnetic field $\mathfrak{5}$. Thus the effect of a transverse field of 26 kilogauss, as expressed by the value of $\left(\mathrm{R}^{\prime}-\mathrm{R}\right) / \mathrm{R}$, was rather more than 5 when the crystalline axis was parallel to the field, and only 0.15 when the axis was normal to the field. To investigate in detail the variation of the change of resistance with the angle between the crystalline and field axes, G I. $12 a$ (G I. 12 shortened and re-coppered) was used. It was remounted and supported in the magnetic field as described above, so that it could be rotated about an axis normal to the field $(a)$ with its length along the axis of rotation, the graphite in this case being always magnetized transversely to its length and the direction of the measuring current, and $(b)$ with its length normal to the axis of rotation.

In the latter case the graphite became successively transversely (cleavage plane normal to the field) and longitudinally magnetized as rotated through $90^{\circ}$.

In such investigations as this it is essential to have the magnetic fields as uniform as possible, and this condition was attained by determining the topography of the field for different distances between the pole end-pieces and adjusting this distance until uniformity was secured *.

* This problem was mathematically discussed by Prof. du Bois, Versl. Kon. Akad. Wet. Amst. xxi. p. 355 (1912); Verh. d. D. Phys. Ges. xiv. p. 758 (1912). 
In case (a) pole end-pieces (VIII.) of frontal diameter $12 \mathrm{~mm}$. were used, and the distance between them found to be very nearly $2 \mathrm{~mm}$. for most uniform field when the current throngh the magnet was sufficient to saturate the iron. This is in good agreement with the theory referred to in the footnote (p. 169). The adjustment can be very exactly made, and finally a field uniform to within less than 1 per cent. was obtained over a transverse range of $8 \mathrm{~mm}$. The

Fig. 2.

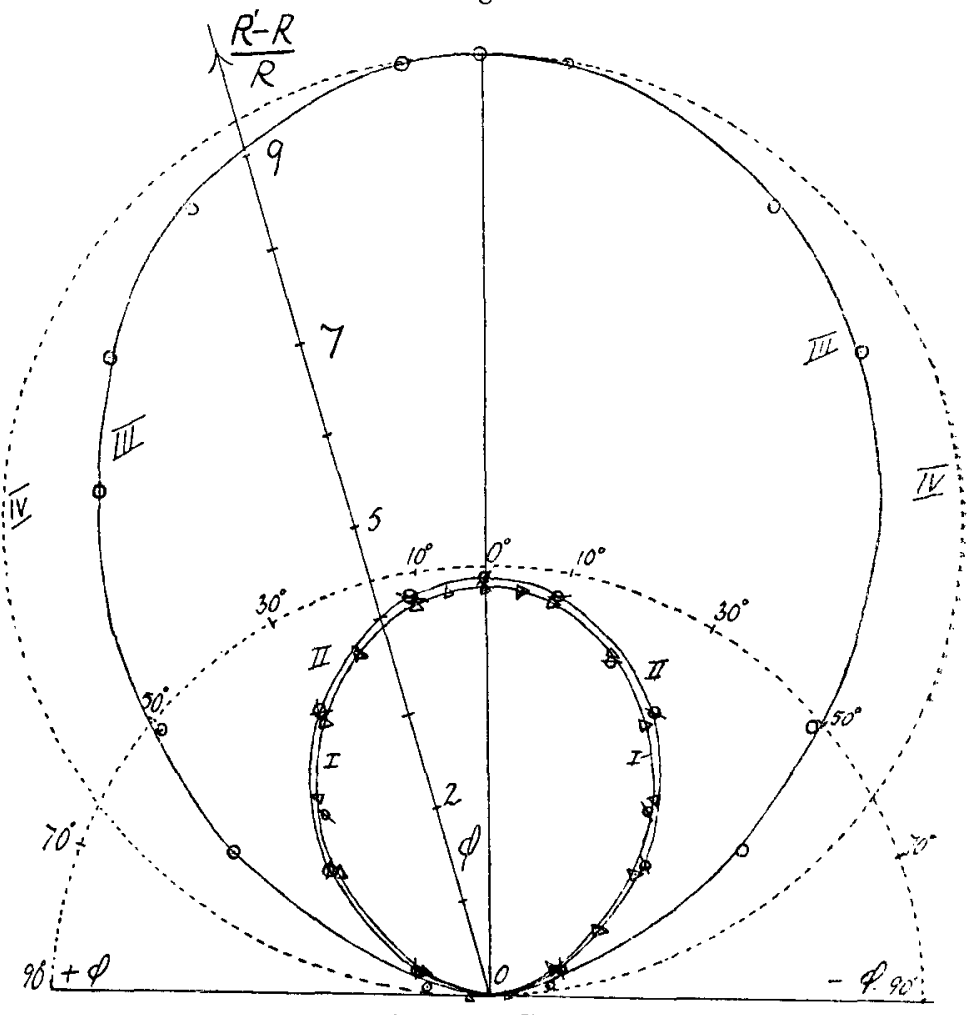

Orientation Curves.

resistance of specimen GI. $12 a$ was determined potentiometrically as before for different inclinations of its crystalline axis to the field as it was rotated through $360^{\circ}$ in uniform transverse fields of 21.8 and $35.2 \mathrm{kgss}$. respectively. The increase of resistance was always positive, being a maximum or a minimum when the cleavage plane was normal or parallel to the field respectively. The results obtained are shown in fig. 2. Curves I. and III. are for a rotation through $180^{\circ}$; 
a further rotation gives again equal corresponding values: $\left(R^{\prime}-R\right) / R$ is plotted radially, the angles $\phi$ shown being those between the field and the crystalline axis,

i.e. $\left(\mathrm{R}^{\prime}-\mathrm{R}\right) / \mathrm{R}=$ the radius vector $\rho=$ function $(\phi)$.

The radius vectors of the curves $I$. and III. represent the effect for the fields $21 \cdot 8$ and $35 \cdot 2 \mathrm{kgss}$. respectively.

Determinations of the change of resistance with magnetic field were also made for the maximum and minimum positions, and are given in Table II. in the second and third columns. The numbers in the second column correspond, mutatis mutandis *, to the curves in fig. 1.

In case (b) no end-pieces were used but only pole-shoes (VII.) of end diameter $43 \mathrm{~mm}$., and at a distance apart of $8.5 \mathrm{~mm}$. The field was practically uniform at this distance. After the graphite had been necessarily differently mounted, the $(b)$ position, the variation of the resistance was determined for $\mathfrak{H}=21.9 \mathrm{kgss}$. as the graphite was rotated from the longitudinal to the transverse position. As the rotation was carried through $360^{\circ}$ practically equal corresponding values were obtained in each quadrant, showing that the increase of resistance is perfectly independent of the direction of the current relative to the field and dependent only on the acute angle between the crystalline and field axes. The results for a rotation of $180^{\circ}$ are shown in fig. 2, curve II. It will be noticed that this curve nearly coincides with curve No. I. ; and remembering the difference in the field (21.9 and 21.8 kilogauss) we may conclude that, within experimental errors, the value of $\left(R^{\prime}-R\right) / R$ depends only on the orientation of the crystalline axis in the field. The variation of $\left(R^{\prime}-R\right) / R$ with longitudinal field is given in Table II., column four.

The results obtained for G I. $12 a$ when transversely magnetized, with the crystalline axis parallel to the field, follow the empirical formula (1) well within experimental errors. $\left(m=1.74^{5} . \quad A=0.0198.\right)$

For the cases when the graphite bas its axis normal to the field and is transversely or longitudinally magnetized the agreement is not so good. The resistances measured and the changes involved are, however, always small, and the experimental errors of greater influence. At the higher

* As the experiments described in this section were carried out at temperatures $\left(19^{\circ}-22^{\circ} \mathrm{C}\right),\left(\mathrm{R}^{\prime}-\mathrm{R}\right) / \mathrm{R}$ has been considered instead of $\left(R^{\prime}-\mathbf{R}\right) / \mathbf{R}_{0}$; the expressions differ only by the constant $1-\frac{\mathbf{R}}{\mathbf{R}_{0}}$, which
differs very little from zero. 
$172 \mathrm{Mr}$. D. E. Roberts on the Effect of Temperature and TABLE II.-G I. 12 a. Temp. $20^{\circ} \mathrm{C}$.

\begin{tabular}{|c|c|c|c|}
\hline \multirow{2}{*}{ jeg kges. } & \multicolumn{2}{|c|}{ Transverse Magnetization. } & \multirow{2}{*}{ 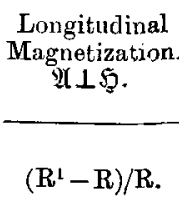 } \\
\hline & $\underset{\left(\mathbf{R}^{\prime}-\mathbf{R}\right) / \mathbf{R} . \overline{\mathfrak{A}} \| \mathfrak{g} .}{\operatorname{Maximum}}$ & $\frac{\text { Minimum }}{\left(\mathbf{R}^{\prime}-\mathrm{R}\right) / \mathrm{R} . \mathfrak{U} \perp \mathfrak{S}}$ & \\
\hline 578 & 040 & $0 \cdot 008$ & $0 \cdot 010$ \\
\hline 960 & 1.04 & $0 \cdot 020$ & 0.020 \\
\hline $14: 78$ & $2 \cdot 16$ & 0.040 & 0.040 \\
\hline $20 \cdot 3$ & $3 \cdot 91$ & 0.070 & 0.075 \\
\hline 21.9 & 456 & 0.083 & 0.082 \\
\hline $23 \cdot 2$ & $4 \cdot 75$ & 0.092 & 0.092 \\
\hline $26 \cdot 15$ & $5 \cdot 91$ & $0 \cdot 115$ & \\
\hline $29 \cdot 75$ & $7 \cdot 36$ & $0 \cdot 148$ & \\
\hline 33.88 & $9 \cdot 28$ & 0.172 & \\
\hline $35 \cdot 2$ & $9 \cdot 89$ & $0 \cdot 189$ & \\
\hline $36 \cdot 4$ & 10.5 & $0 \cdot 194$ & \\
\hline
\end{tabular}

fields the agreement is better. In the orientation experiments, assuming formula (1) to be true, it is reasonable to expect that $\left(R^{\prime}-R\right) / R$ should now be given by an equation such as

$$
\frac{\mathbf{R}^{\prime}-\mathbf{R}}{\mathbf{R}}=\mathbf{A} \mathfrak{S}_{\|}^{m}+\mathrm{A}^{\prime} \mathfrak{W}_{\perp}^{m^{\prime}}
$$

where $\mathfrak{D}_{\|}$denotes the component of the field along the axis, and $\mathfrak{S}_{\perp}$ that at right angles.

Or

$$
\frac{\mathrm{R}^{t}-\mathrm{R}}{\mathrm{R}}=\mathrm{A}(\mathfrak{5} \cos \phi)^{m}+\mathrm{A}^{\prime}(\mathfrak{S} \sin \phi)^{m^{\prime}},
$$

and this, since $\mathfrak{W}$ is kept constant, may be written

$$
\frac{\mathrm{R}^{\prime}-\mathrm{R}}{\mathrm{R}}=2 a \cos ^{m} \phi+2 a^{l} \sin ^{m^{\prime}} \phi . \quad \text {. . . . }
$$

Here $2 a$ and $2 a^{\prime}$ are constants, and are experimentally determined when $\phi=0$ or $\pi / 2$ respectively.

Applying equation (2) to the experimental points given in fig. 2 , curve III., putting $2 a=9 \cdot 80$ and $2 a^{\prime}=0 \cdot 202$, it was first found that putting $m=m^{\prime}=1 \cdot 74^{5}$, fair agreement between the calculated and the observed values for intermediate values 
of $\phi$ were obtained. Now the second term in equation (2) is, except when $\phi$ is nearly $90^{\circ}$, very small compared with the first term. One is therefore led to think that possibly it may represent nothing more than a spurious effect due to the crystalline non-uniformity of the samples; in fact, deviations of the axes at different points of the graphite strip of the order of $1^{\circ}$ would suffice to explain such an apparent effect; the distribution of the current may also be nonunidirectional. It is hardly possible with the graphite specimens yet available to settle this point. If we may neglect the second term, we obtain the equation in polar coordinates

$$
\frac{\mathrm{R}^{\prime}-\mathrm{R}}{\mathrm{R}}=\rho=2 a \cos ^{m} \phi . . \quad \text {. . . . }
$$

The curves in fig. 2 are drawn according to this equation, $a$ being of course determined by experiment. The points indicated were experimentally determined. Curve IV. is the circle

$$
\rho=2 a \cos \phi
$$

\section{Experiments at Low and High Temperatures.}

In these experiments the cleavage plane of the specimen investigated was kept normal to the tield. Observations were taken at temperatures of $-179^{\circ}, 0^{\circ},+11^{\circ},+95^{\circ},+179^{\circ} \mathrm{C}$., the field being varied from 0 to $40 \mathrm{kgss}$. The methods of measurement were the same as those adopted for the measurements at ordinary temperatures ; the determinations offered no difficulties, the resistances being quite steady. At the lower and higher temperatures thermo-electric effects were sometimes evident, but by successive reversals these were eliminated*. For the specimens G I. and IV. investigated an increase of resistance with temperature was found, the coefficient being of the high order of +0.001 per degree. The temperature curve $(\mathfrak{W}=0) \mathrm{R} / \mathrm{R}_{0}=\mathrm{Funct}(\theta)$ is given in fig. 3 for specimen GI. 15 ; a similar curve (also shown) was obtained for GI. 11. Both show a strongly marked inflexion in the region of ordinary temperatures. It is interesting to compare these with one obtained by Kamerlingh Onnes and Clay $\dagger$, which is for a bismuth spiral,

* It was incidentally observed that these thermo-electric effectswhen occurring at the connexions of the graphite and therefore within the magnetic field-were influenced by the field. Thus in one case the thermo-electric offect was increased fourfold by a field of $26 \mathrm{kgss}$.

$+\mathrm{H}$. Kamerlingh Onnes and J. Clay, Versl. Kon. Akad. Wet. Amst. xvi. p. 176 (1907). Cf. also W. Nernst and F. A. Lindemann, Berl. Ber. p. 306 (1911). 
174 Mr. D. E. Roberts on the Effect of Temperature and

No. 301, by Hurtmann and Braun, and extends to $-259^{\circ} \mathrm{C}$.; this shows in the neighbourhood of $-200^{\circ}$ a weak extended inflexion; this curve is given in fig. 3 , and is denoted by Bi.

Fig. 3.

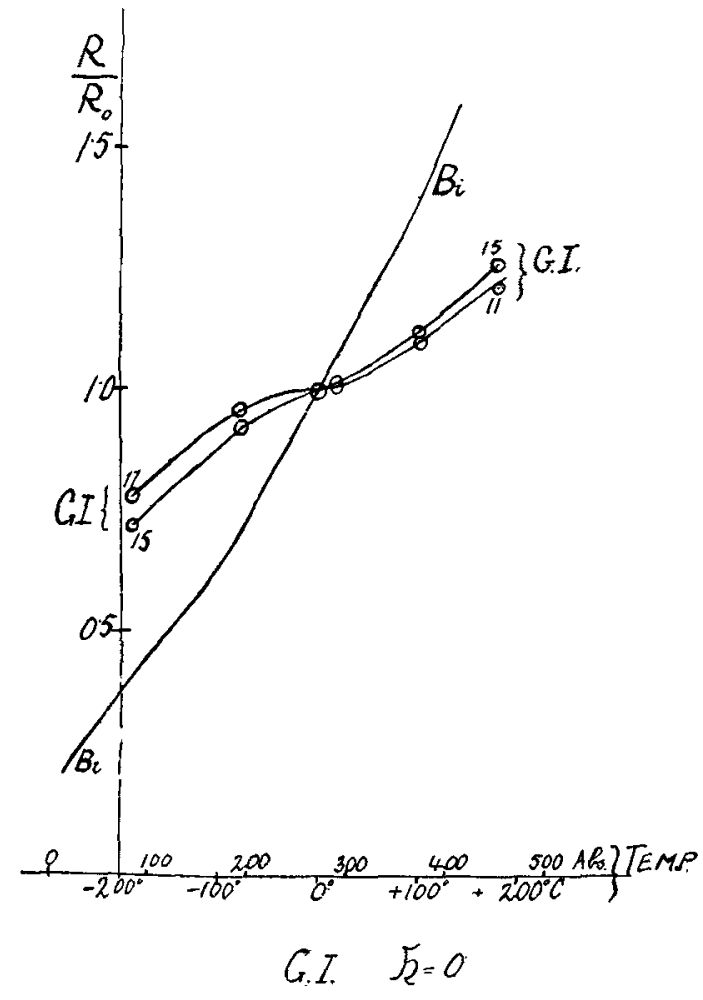

All the samples tested of the variety G I. behaved similarly although exhibiting appreciable quantitative differences, as is shown by the values of $R_{18} / R_{0}$ in Table $I$. Moreover, sometimes changes in $R_{18}$ or $K_{0}$ were observed after the graphite had been subjected to extreme temperatures.

The temperat: re during a series of radings, the graphite being between the poles of the e'ectromagnet, was determined as follows. Hefore commencing, the current required to be sent through the electromagnet in the reverse direction in order to reduce the residual field to zero was determined. Then, to measure the temp rature, the graphite being in position, this reverse current was set up and the resistince of the graphite found. 'The temperature of the graphite 
was then deduced from the temperature curve $(\mathfrak{5}=0)$ fig. 3 . Owing to the difficulty of exactly getting rid of the residual field without setting ap a field in the opposite direction, and on account of the small change of resistance with temperature, this method of determining the temperature does not seem to be capable of great accuracy. The isothermals at low and high temperatures were determined for G I. 11 and G I. 15. Except for the difference in the magnitude of the changes of resistance concerned similar results were found. The results obtained with specimen G I. 15 are shown as

Fig. 4.

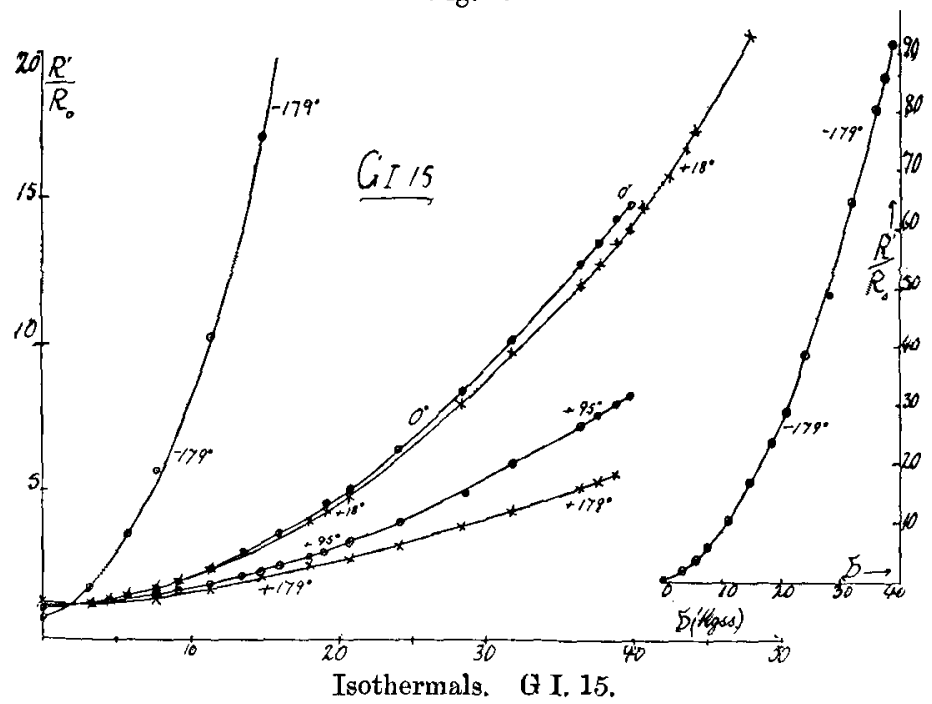

isothermal curves (fig. 4), from which the so-called isopedic curves $(\mathfrak{D}=$ constant) may easily be deduced. As will be seen, the increase of resistance is much greater at low temperatures. At the temperature of liquid air the increase is 9300 per cent. for a tield of 38.8 kilogauss, the increase at $18^{\circ}$ (. in the same field being 1250 per cent.

The isothermal curves for the lower temperatures cannot be represented by an equation of the form (1) : at higher tempiratures this seems to be the case, although more accurate measurements appear desirable. I regret that owing to external circumstances I was unable to repeat the measurements at different temperatures, so that this section of the research can hardly be regarded as completed. As regards the practical application of the large effect here 
found in graphite to the measurement of magnetic fields, it appears that the lack of definition and mechanical strength of this material would form serious objections. Also the resistance of a graphite piece is much less than that of a bismuth spiral. The chief disadvantage in the use of the latter lies in the difficulties of determining the temperature correction; the isothermals intersect one another; this, however, holds also in the case of graphite, as is seen by referring to fig. 4 .

I beg, in conclusion, to express my gratitude to Prof. $\mathrm{du}$ Bois, in whose laboratory the experiments were carried out, for his constant advice and criticism, and for his kindness in placing the powerful du Bois electromagnet and other apparatus at my disposal. I am also indebted to Dr. W. J. de Haas for many valuable suggestions during the course of the investigation.

Bosscha-Laboratorium, Berlin.

January 3rd, 1913.

VII. On the Use of the Interferometer for the Study of Band Spectra. By R. W. Wood, Professor of Experimental Physics, Johns Hopkins University *.

[Plate IV.]

TN a paper published in 1907 (Phys. Zeit. viii. p. 607) 1 I drew attention to peculiar geometrical arrangements of the Fabry and Perot interference fringes exhibited in photographs of the titanium spectrum made by Dr. Pfund. As I showed in this paper, the existence of a symmetrical pattern in such a photograph indicated that the lines were spaced according to some mathematical law, and I suggested that in this way regularities might be found in very complicated line spectra.

The photograph in question was made by passing the light from the titanium are through the half-silvered plates of a Tabry and Perot interferometer, and focussing an image of the circular ring system upon the slit of a grating spectrograph, the method being the one universally adopted for the determination of wave-lengths by interference methods. Owing to the large difference of path between the interfering streams of light, the order of the fringes changes by a considerable number as we pass from one line to the next, even in the case of lines lying very close together. If the

* Communicated by the Author. 